\title{
The Bionic Water Channel of Ultra-Short, High Affinity Carbon Nanotubes with High Water Permeability and Proton Selectivity
}

\author{
Guangli Liu ${ }^{1,2}$, Bin Zhou ${ }^{3,4}$, Jinwei Liu ${ }^{1,2}$ and Huazhang Zhao ${ }^{1,2, *}$ \\ 1 Department of Environmental Engineering, Peking University, Beijing 100080, China; \\ luckydog27227@163.com (G.L.); liujinwei@pku.edu.cn (J.L.) \\ 2 The Key Laboratory of Water and Sediment Sciences, Ministry of Education, Beijing 100871, China \\ 3 Department of Bioengineering, Qilu University of Technology (Shandong Academy of Sciences), \\ Jinan 250301, Shandong, China; 17862972742@163.com \\ 4 State Key Laboratory of Biobased Material and Green Papermaking, Jinan 250353, Shandong, China \\ * Correspondence: zhaohuazhang@pku.edu.cn; Tel.: +86-10-62758748
}

Citation: Liu, G.; Zhou, B.; Liu, J.; Zhao, H. The Bionic Water Channel of Ultra-Short, High Affinity Carbon Nanotubes with High Water Permeability and Proton Selectivity. Sustainability 2021, 13, 102. https://dx.doi.org/ $10.3390 /$ su13010102

Received: 12 November 2020 Accepted: 18 December 2020 Published: 24 December 2020

Publisher's Note: MDPI stays neutral with regard to jurisdictional claims in published maps and institutional affiliations.

Copyright: () 2020 by the authors. Licensee MDPI, Basel, Switzerland. This article is an open access article distributed under the terms and conditions of the Creative Commons Attribution (CC BY) license (https: / / creativecommons.org/ licenses/by/4.0/).

\begin{abstract}
The development of the bionic water channel aims to replace the possible use of natural aquaporins (AQPs) for water purification, while retaining the ability of natural AQPs to carry out ultra-fast water transport and repel ions. Carbon nanotube channels (CNTCs) are a convenient membrane-based model system for studying nano-fluidic transport that replicates a number of key structural features of biological membrane channels. In this report, we describe protocols for CNTCs synthesis by ultrasound-assisted cutting of long CNTs in the presence of lipid amphiphiles. CNTCs have a similar thickness to the lipid membrane and high affinity for it. The ultra-short highaffinity CNTCs have high permeability and ion selectivity. The water permeability of the CNTCs is $1936 \pm 123 \mu \mathrm{m} / \mathrm{s}$, which is 2.3 times that of natural AQPs, and completely rejects salt ions. In general, carbon nanotubes represent a multifunctional nanopore building module for creating high-ranking functional bionic materials. This study has reference significance for the design of new bionic water channel and the actual development of bionic membrane based on CNTs.
\end{abstract}

Keywords: water purification; AQPs; bionic water channel; ultrasonic cutting; carbon nanotubes

\section{Introduction}

The shortage of freshwater resources poses a huge threat to the sustainable development of human society. It has affected most parts of the world and will be exacerbated by population growth and environmental pollution at the end of this century [1]. Membrane water treatment technology has an important contribution to low-carbon sustainable development. Water purification and membrane desalination technologies are effective means to increase the supply of fresh water. Membrane water treatment technologies represented by reverse osmosis and nanofiltration processes are often used in the treatment of daily drinking water, seawater desalination, and high-purity industrial water production $[2,3]$. Yet, nanofiltration and reverse osmosis are still high-energy-consuming procedures with low water permeability [4]. The ideal water treatment membrane should have high water permeability, excellent desalination ability, and minimum driving force [5].

Bionic membranes based on Aquaporins (AQPs) have a promising future in the field of water purification. Natural AQPs have a special pore structure with high selectivity and permeability to water, and their characteristics provide new insights for the construction of highly selective and permeability water treatment bionic membranes. By imitating the composition and structural layout of cell biofilms and learning from the separation and permeation mechanism of natural cell biofilm, researchers have embedded AQPs into the base membrane material to construct a bionic membrane with specific selectivity and high permeability to water [6]. Bionic membrane based on AQPs has become a research 
hotspot as an efficient pure water recovery membrane technology [7]. However, AQPs biomimetic membranes face the problems of protein inactivation and poor selectivity in actual operation. The emergence of bionic water channels provides a good idea to solve the problems of AQPs inactivation and poor selectivity [8-10].

Common bionic water channels include carbon nanomaterial water channels [11,12], organic water channels [13,14], peptide pore water channels [15], etc. These artificial bionic water channels are characterized by their easy formation of transmembrane structures and their narrow pore size. Aquaporins (AQPs) in bionic membranes are transmembrane proteins accurately designed by nature with high permeability and selectivity to water [16,17]. Imitating the structure of natural AQPs to build a bionic water channel can solve the problem of deactivation and poor selectivity of natural AQPs and develop new water purification materials $[18,19]$. Developing bionic water channels with special pore structures, specific selectivity, and highly efficient water permeability to imitate or replace AQPs is one of the current research directions in water purification technology. There are great breakthroughs in structure and function of this special type of protein variant and its water/solute selectivity is close to perfect. However, it is quite different from natural AQPs that have special pore channels that allow selective water transport and solute rejection. The ideal bionic water channel should have a channel structure that can be easily formed during the preparation process and can provide high-efficiency water delivery and a high-affinity outer surface that is compatible with the lipid membrane environment [20]. Researchers' interest in bionic water channels has increased in recent years [21]. However, there are still two basic problems:(i) In the process of preparing the bionic water channel, the preparation length cannot reach the thickness of the lipid film, which further affected its specific selectivity and efficient permeability to water; (ii) during the preparation process, it is difficult to control the optimization of the preparation conditions to obtain the bionic water channels with good biocompatibility in a lipid membrane environment.

The emergence of carbon nanotubes (CNTs) provides alternative materials for the design of high-performance bionic water channels [22]. CNTs are widely used in the field of water treatment biomimetic membranes due to their inherent nanometer size and hydrophobic characteristics of the tube wall $[23,24]$. CNTs with efficient water transport performance and adjustable selectivity potential have bright application prospects in the field of water purification [25]. The studies of CNTs water channel mostly focus on imitating the form of pores, but few on the CNTs' preparation length, the influence of CNTs' length on water permeability, and their compatibility with a lipid membrane environment. These are the key factors preventing the development of CNTs water channels. In this paper, a cuttingedge and environmentally friendly ultrasonic technology with high efficiency was used to cut raw carbon nanotubes. Natural active agent-assisted ultrasonic cutting technology is used to prepare CNTs that are similar to the thickness of the phospholipid membrane and with high phospholipid affinity. It acts as a water channel together with natural AQPs in the liposome system. We also studied water permeability and ion selectivity of the novel CNTs based water channel. This paper provides unique insights and ideas for designing a new type of bionic water channel.

\section{Experiment Section}

\subsection{CNTCs Prepared by Ultrasonic Cutting}

For a typical ultra-short CNTCs preparation procedure [26], $2 \mathrm{~mL}$ lipid suspension (10 mg/mL DOPC in chloroform) was added to a $20 \mathrm{~mL}$ glass vial, and the solvent was evaporated using a steady stream of argon gas or air to form a lipid film on the walls of the glass vial; it was further dried in a desiccator overnight to remove trace solvent residue. Then, $2 \mathrm{mg}$ (1.5 $\mathrm{nm}$ CNTs) was purified in a thermogravimetric analysis (TGA) system. The maximum baking temperature for $1.5 \mathrm{~nm} \mathrm{CNTs}$ was set to $461.5^{\circ} \mathrm{C}$. Fourteen $\mathrm{mL}$ of Milli-Q water was added to the purified CNTs, and that mixture was added to the corresponding dried lipid film. The CNT/lipid mixture was first bath-sonicated for $20 \mathrm{~min}$ to disperse the CNTs in lipid solution. This is followed by probe-tip sonication at $200 \mathrm{~W}$ 
power for $36 \mathrm{~h}$. The sonication program was run in $3 \mathrm{~s}$ pulses with $1 \mathrm{~s}$ pause between each pulse. The ultrasonication converter head needs to be cooled to avoid overheating during operation. To prevent overheating of this component, we directed a constant stream ( $15 \mathrm{psi}$ ) of cool, dry air directly onto the convertor head. In order to keep the sample from overheating, we placed the sample vial into a copper water circulating block that constantly cycles $30^{\circ} \mathrm{C}$ coolant through the block to maintain a constant chamber/sample temperature during the 36 hours sonication procedure. To purify the shortened CNTCs from the uncut CNTs, the sonication-processed solution was transferred to a $15 \mathrm{~mL}$ conical centrifuge tube and centrifuged at $10,000 \mathrm{rpm}$ for $1 \mathrm{~h}$ at $21^{\circ} \mathrm{C}$ followed by a 20,000 rpm for $10 \mathrm{~min}$ at $21^{\circ} \mathrm{C}$. After centrifugation, the light-gray supernatant containing the CNTC solution was carefully aspirated with a glass pipet and stored at $4{ }^{\circ} \mathrm{C}$. The pellet fraction was discarded. When exploring the effects of different types of surfactants on the synthesis of ultra-short CNTs, the operation process is the same as the previous article, replacing DOPC with monolauryl phosphate $(\mathrm{ROPO}(\mathrm{OH}))_{2} \mathrm{R}$ or laureth phosphate $\left(\left[\mathrm{RO}\left(\mathrm{CH}_{2} \mathrm{CH}_{2} \mathrm{O}\right)_{n}\right]_{2} \mathrm{PO}(\mathrm{OH})\right)$ was tested separately.

\subsection{UV-vis-NIR Spectrum of CNTCs}

To sonicate, $0.5 \mathrm{mg}$ CNTCs was dissolved in $1 \mathrm{~mL} 1 \%$ SDS-containing $\mathrm{D}_{2} \mathrm{O}$ solution for $1 \mathrm{~h}$ and then corner tip sonication at $21 \mathrm{~W}$ was performed for $30 \mathrm{~min}$ to prepare a solution for fluorescence spectroscopy. The sample was purified by centrifuging the solution at 10,000 rpm for $20 \mathrm{~min}$. The supernatant was decanted, and the resulting CNTCs dispersion was used for emission scanning. The excitation wavelength range used for typical measurement is $500-800 \mathrm{~nm}$, the emission limits are $900-1450 \mathrm{~nm}$, the slit width is $4 \mathrm{~nm}$, and the combination time on the spectrofluorometer is $30 \mathrm{~s}$.

\subsection{Dynamic Light Scattering Test the Particle Size of CNTCS}

The average particle size of ultra-short carbon nanotubes was determined by operating Zetasizer Nano ZS (Malvern, UK) at $22 \pm 1{ }^{\circ} \mathrm{C}$. Fifty $\mu \mathrm{L}$ of carbon nanotube sample was added into an available volume cuvette and the light scattering intensity of the carbon nanotubes was measured. Each size's reading is the average of 10 independent measurements. The measured result was used for permeability calculation and to determine whether it was the target diameter we needed.

\subsection{Raman Spectra of CNTCs}

A mini-Raman spectrometer was used to measure the Raman spectra of CNTs samples at wavelengths of 473 and $633 \mathrm{~nm}$. The laser energy used to study the CNTs hole was set below $100 \mathrm{~W} / \mathrm{cm}^{2}$ to prevent the sample from heating. In order to collect samples for Raman analysis, a $150 \mu \mathrm{L}$ aliquot of the CNTs aqueous dispersion was dried in a vacuum dryer on a glass slide for about $3-4 \mathrm{~h}$ to form spots on the glass slide, and then the measurement was performed in the spectrometer. The Raman spectra was normalized to G-band intensity for comparison between different samples. Parameter settings: sample exposure time was 16 seconds, laser wavelength was $633 \mathrm{~nm}$, laser power was $13 \%$, aperture pinhole was $50 \mu \mathrm{m}$, grating position was set to single, collection range was 3925 to $96 \mathrm{~cm}^{-1}$, and center display was $2284 \mathrm{~cm}^{-1}$.

\subsection{Water Permeability Test of CNTCs}

Water permeabilities of $1.5 \mathrm{~nm}$ CNTCs or pure liposomes were measured using a stopped-flow instrument at $22 \pm 1.5^{\circ} \mathrm{C}$. The liposome samples were rapidly mixed with a hypertonic solution that caused them to shrink due to the osmotic gradient driving a water efflux. According to Rayleigh-Gans-Debye (RGD) light scattering theory, the sudden change of fracture volume leads to the increase of $90^{\circ}$ light scattering. All osmotic pressure differences are measured experimentally on a freezing point osmometer. Osmotic pressure difference between inside and outside vesicles and the water permeability of the vesicles can make the water molecules transport outward, resulting in the decrease of the volume 
of the vesicles. A classical experiment for permeability testing involves mixing liposomes (5 mg/mL DOPC) with an osmotic agent buffer at a ratio of 1:7 (40 $\mu \mathrm{L}: 280 \mu \mathrm{L})$. We tested each concentration for 5-10 times. Exponential decay Equation (1), where $\mathrm{Y}$ is the intensity of the light scattering signal, $\mathrm{A}$ is a negative constant, $\mathrm{t}$ is the recording duration, and an exponential coefficient describing the change of light scattering is $\mathrm{k}$. Its value is obtained by fitting the stopped flow result curve to a single-order index (Fluorescence $=\mathrm{a}^{*} \mathrm{e}^{-\mathrm{k}^{*} \mathrm{t}}+\mathrm{c}$, where a and $c$ are fitting parameters). The final water permeability $\left(P_{f}\right)$ of the vesicle is calculated by Equation (2). $S / V_{0}$ is the ratio of the surface area of the vesicle to the initial volume, $V_{w}$ is the partial molar volume of water $(0.018 \mathrm{~L} / \mathrm{mol})$, and $\Delta o s m$ is the osmotic pressure difference between the aqueous solution inside and outside the vesicle.

$$
\begin{aligned}
& Y=A \exp (-k t) \\
& P_{f}=\frac{k}{\frac{S}{V_{0}} V_{w} \Delta_{o s m}}
\end{aligned}
$$

The reflection coefficient $\sigma$ is determined by analyzing the permeability of specific absorbed solute and reference solute under the same $\Delta o s m$. In this study, the relatively large sucrose molecule is the reference solute, which is almost completely rejected by liposomes vesicles [4]. Thus, the apparent reflection coefficient of $\mathrm{NaCl}$ is calculated by the following formula:

$$
\partial=\frac{P_{f, \text { colute }}}{P_{f, \text { reference }}}
$$

In order to determine the activation energy of water permeability, the experiment was repeated to stop the flow under different temperature conditions (10 to $40{ }^{\circ} \mathrm{C}$ ), and the exponential rise rate calculated from the experiment was plotted against the reciprocal of the temperature to determine the Arrhenius activation energy.

\subsection{Ion Selectivity Test of CNTCs}

Preparation of liposomes containing fluorescent dyes (HPTS): $10 \mathrm{mM}$ DOPC lipid with $10 \%$ cholesterol were dissolved in chloroform to a concentration of $5 \mathrm{mg} / \mathrm{mL}$ [27]. A rotary evaporator was used to evaporate $1 \mathrm{~mL}$ of DOPC solution in a $5 \mathrm{~mL}$ round bottom flask to form a lipid membrane. The lipid membrane was dried in vacuum at least $2 \mathrm{~h}$ to evaporate the remaining chloroform. Using HEPES buffer solution (2 mL, KCl (100 mM), HEPES (10 mM), pH 7.0) containing HPTS (0.1 mM) hydrated at $40{ }^{\circ} \mathrm{C}$ for $2 \mathrm{~h}$ to obtain a milky suspension. The sample was put in liquid nitrogen $\left(-196^{\circ} \mathrm{C}\right)$ and frozen for $3 \mathrm{~min}$, then transferred to a $50{ }^{\circ} \mathrm{C}$ water bath for $3 \mathrm{~min}$. This process was reciprocated 10 times. The suspension was dialyzed three times with a membrane tube $(\mathrm{MWCO}=14,000)$ to remove the unembedded residual dye and produce a vesicle suspension. The obtained sample was subjected to extruder filtration 21 times with a $400 \mathrm{~nm}$ polycarbonate filter membrane to finally obtain a concentration of $10 \mathrm{mM}$ DOPC liposomes with embedded fluorescent dye. The sample was stored at $80{ }^{\circ} \mathrm{C}$ for later use.

Fluorescence experiment: HEPES buffer solution $(2.0 \mathrm{~mL}, \mathrm{HEPES}(10 \mathrm{mM}), \mathrm{NaCl}$ $(100 \mathrm{mM}), \mathrm{pH}=5.6)$ was placed in a fluorescent cuvette. The mixed solution (including vesicle suspension $(10 \mathrm{mM}, 100 \mu \mathrm{L})$, CNTCs channel solution $(1.0 \mathrm{mM})$, and valinomycin $(1.2 \mu \mathrm{M}))$ dissolved in DMSO $(30 \mu \mathrm{L}))$ was added in a cuvette with gentle stirring. Fluorescence intensity (It) was continuously monitored at $510 \mathrm{~nm}$ (460 nm excitation) for $10 \mathrm{~min}$. Then, $10 \mu \mathrm{L}$ of $50 \%$ Triton ${ }^{\circledR} \mathrm{X}-100$ solution was added while slowly stirring. The fluorescence intensity was monitored until there was no change. Finally, the data was normalized, and the fractional change in fluorescence intensity was obtained by $\left(\mathrm{I}_{t}-\mathrm{I}_{0}\right) /\left(\mathrm{I}_{\infty}-\mathrm{I}_{0}\right)$, where $I_{0}$ is the initial fluorescence intensity. 


\section{Results}

\subsection{CNTCs Synthesis}

The synthesis process of CNTC is to disperse CNT bulks into single carbon nanotubes. It was cut into biocompatible sizes (10-15 nm) and kept stable in watery solution. In order to diminish the contamination of the sample, the oxidation temperature was determined by using the TGA system to remove impurities in the CNT material. The initial oxidation temperature of carbon nanotubes with a diameter of $1.5 \mathrm{~nm}$ was $461.5^{\circ} \mathrm{C}$, which is consistent with the report [28]. In the synthesis process, cutting-edge ultrasound technology is mainly used for CNT splitting. It is easy to dissolve the purified CNT material through gentle ultrasonic treatment, and the lipid molecules were adsorbed on the surface of the CNT and used as a surfactant. As shown in Figure 1, long-term ultrasonic treatment of the microtip caused the microbubbles near the surface of the CNT to cavitate and collapse and gradually broke the macroscopic nanotubes into shorter fragments; this process was repeated throughout the ultrasound treatment $[29,30]$.

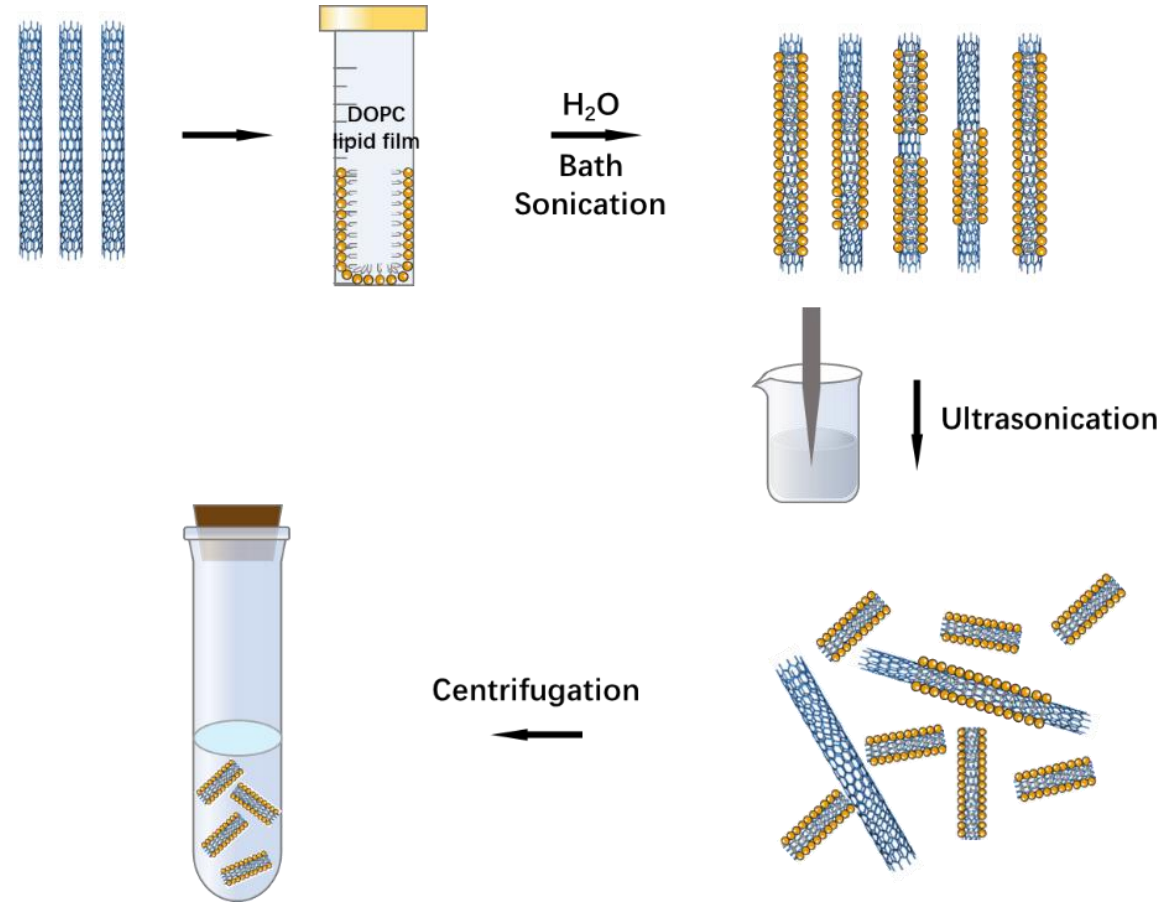

Figure 1. Schematic diagram of ultra-short and high-affinity CNTC preparation.

In order to determine the relationship between the cutting time and the required CNTs length, we first determined the best cutting power (See Figure S1 for details). The dynamic size distribution in the sample is measured by sampling at intervals. Dynamics (Figure 2a) showed that the average particle size of the sample was decreasing, and there was no significant change in particle size after $24 \mathrm{~h}$. Therefore, the cutting work was basically completed at $24 \mathrm{~h}$. Figure $2 \mathrm{~b}$ shows the particle size close to the thickness of the lipid film in $24 \mathrm{~h}$. The purification step removed uncut CNTs and aggregated CNTCs. The resulting CNTCs solution was light gray (as illustrated in Figure 2a) and was stored at $4{ }^{\circ} \mathrm{C}$. After being completely dried and then resuspended in an aqueous solution by ultrasonic treatment, this process showed that the liposome coating was stably attached to the CNTs surface. Carbon nanotubes encapsulated in amphiphilic lipid molecules in the solution retained most of the optical properties of carbon nanotubes. The absorption spectra of all lipid carbon nanotube complexes (Figure $2 c$ ) have the characteristics of $E_{22}$ and $E_{11}$ optical transitions [31]. The quantum imprisonment was increased along the axle of the carbon nanotubes, which resulted in a blue divert in absorbance for shorter carbon nanotubes [32]. We found the blue divert of the UV-Vis-NIR absorption spectrum of CNTCs (Figure 2c), 
which confirmed that sonication caused the nanotubes to shorten significantly. In the Raman spectrum, a peak of $2800 \mathrm{~cm}^{-1}$ was used as the characteristic liposomes signal. The original carbon nanotube material (Figure 2d) showed the key signals of the D-band at $1310 \mathrm{~cm}^{-1}$, G-band at $1590 \mathrm{~cm}^{-1}$, and G'band at $2600 \mathrm{~cm}^{-1}$, respectively. The Raman spectrum of DOPC liposomes showed a wide signal across the whole spectrum and a peak signal (L-band) at $2800 \mathrm{~cm}^{-1}$ (Figure 2d), which is similar to previous reports [33]. The range between $2800 \mathrm{~cm}^{-1}$ and $3100 \mathrm{~cm}^{-1}$ corresponds to C-H bond stretching. The L-band signal intensity was equivalent to the Raman signal of the lipid molecule, the CNTCs with high activity had a much stronger signal than the CNTCs with low activity. The processed and centrifuged CNTCs sample contains very few free lipids, and the L peak in the highly active CNTCs spectrum comes from the liposomes adsorbed on the appearance of the nanotubes. In contrast, the smaller intensity of the lipid spectrum combined with the stronger G-band indicates poor lipid coating, resulting in a decrease in CNTCs activity (incorporation efficiency) (See Figure S2 for details). This observation was consistent with our subsequent test results.

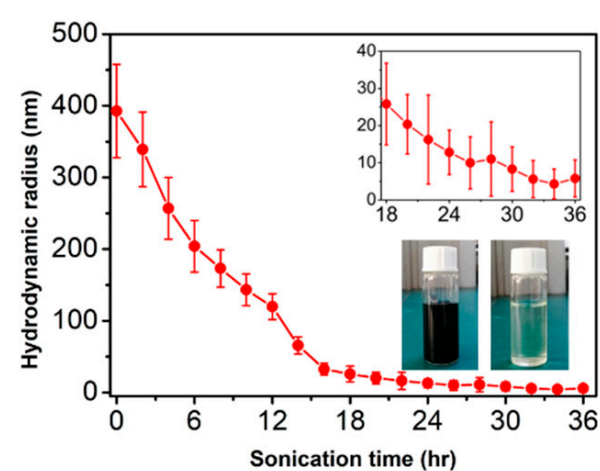

(a)

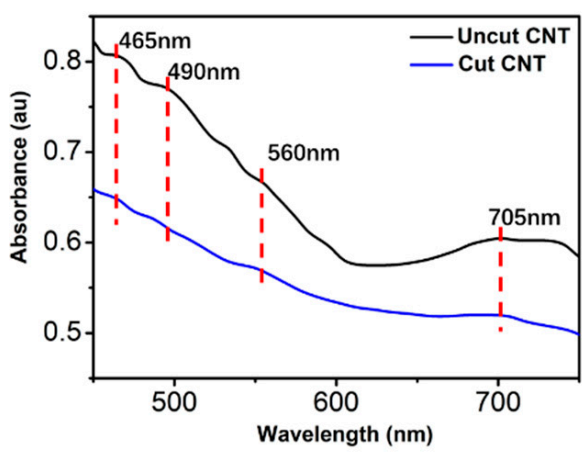

(c)

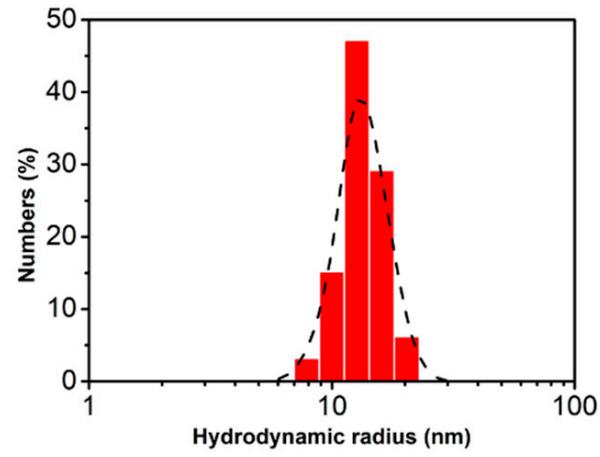

(b)

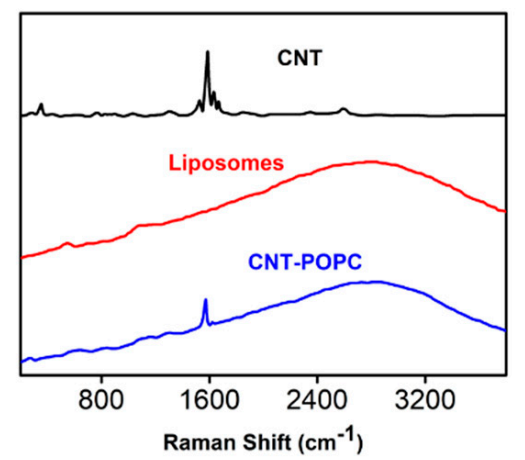

(d)

Figure 2. Preparation and characterization of ultra-short and high-affinity CNTCs. (a) The relationship between the average particle size of CNTs and ultrasonic time. Photographs of CNTCs dispersions separated by centrifugation before and after cutting. (b) Kinetic particle size of ultrashort CNTCs sample. (c) The UV-vis-NIR absorption spectrum of CNTCs before and after cutting. The dotted line indicates the position of the main spectral features. (d) Purified CNTCs raw materials (black curve), characteristic signal peaks of DOPC (red curve), and high-yield lipid-coated CNTCs samples.

\subsection{Characterization of Water Permeability}

We investigated the permeability of different CNTCs spontaneously inserted into lipid membranes to form transmembrane channels [34] to water and ions, including CNTCA ( $\sim 30 \mathrm{~nm}$ long CNTs fragment, See Figure S3 for details), CNTCB ( 12 nm long CNTs fragment), and high-affinity CNTCC ( $12 \mathrm{~nm}$ long CNTs fragments). The liposomes and CNTC-liposomes (Figure 3a) were exposed to a hypertonic buffer solution containing sucrose permeate to measure the water permeability of CNTC in a stopped-flow instrument 
(See Figure 44 for details). Figure $3 \mathrm{~b}$ shows the light scattering curves of several types of water channels (See Figure S5 for details). We calculated the water permeability of CNTCs under neutral conditions by light scattering curve; the water permeability of CNTCC was $1936 \pm 123 \mu \mathrm{m} / \mathrm{s}$, which was 2.3 times that of natural AQPs (the water permeability of natural AQPs we obtained was consistent with related reports [15], See Figure S6 for details). The narrow CNTCs force the cluster water in the water to become a one-dimensional (1D) waterline and quickly pass through the narrow hole $[35,36]$. The permeability of CNTCA and CNTCB were lower than CNTCC, which are $433 \pm 43.8 \mu \mathrm{m} / \mathrm{s}$ and $1275 \pm 81.6 \mu \mathrm{m} / \mathrm{s}$, respectively. This verifies that the length of CNTCs determines their water permeability; the closer the length is to the thickness of the lipid membrane, the better the water permeability. Our high-affinity CNTCs prepared by lipid encapsulation have high insertion efficiency, which has been reflected by water permeability. Figure $3 c$ shows the water permeability of several types of water channels under different $\mathrm{pH}$ conditions, with the highest permeability occurring near neutral conditions. For AQPs, with the increase of $\mathrm{H}^{+}$concentration, permeability greatly decreases when the $\mathrm{pH}$ value is 5.5 , and permeability increases again in a strong acidic environment with a $\mathrm{pH}$ value of less than 3.5. On the other hand, with the increase of the $\mathrm{OH}^{-}$concentration in the vesicle solution, the permeability value greatly decreased at $\mathrm{pH} 9$ and then increased slightly in a strong alkaline environment with a $\mathrm{pH}$ greater than 10 . This indicates that the water filtration mechanism of AQPs is affected by $\mathrm{H}^{+}$and $\mathrm{OH}^{-}$. Interestingly, the water permeability of different CNTCs were almost the same as that of neutral conditions, whether in a strong acid environment or a strong alkali environment. Changing in $\mathrm{pH}$ did not affect their water permeability, which is mainly because CNTCs channel lacks the internal functional amino acids of natural aquaporin [37,38]. Figure $3 \mathrm{~d}$ shows the $\mathrm{NaCl}$ reflection coefficient of liposomes and CNTC-liposomes (Equation (3), sucrose is used as a reference solute [7]). In the presence of different solutes, the obvious reflection coefficient of $\mathrm{NaCl}$ is close to 1. This shows that DOPC lipid bilayers, AQPs, and CNTCC have good $\mathrm{NaCl}$ rejection. The selectivity of CNTC to cation and anion was studied by base pulse experiment. The $\mathrm{pH}-$ sensitive dye HPTS [39] was captured in the liposome cavity with a diameter of $400 \mathrm{~nm}$ (See Figure S7 for details); the $\mathrm{pH}$ in the liposome cavity was 7 . The $\mathrm{pH}$ gradient of 0.6 units was increased externally by adding $\mathrm{NaOH}$ solution. At $200 \mathrm{~s}, \mathrm{NaOH}$ solution was added and Triton ${ }^{\circledR} \mathrm{X}-100$ was added at $1600 \mathrm{~s}$, which dissolved liposomes and caused the $\mathrm{pH}$ gradient to collapse completely. The change of the transmembrane $\mathrm{pH}$ gradient means that the solution in the liposome cavity was alkalized, and then the signal was sent out by the change of HPTS fluorescence. The direct information transported by $\mathrm{H}^{+}$(efflux) or $\mathrm{OH}^{-}$(influx) reflects the indirect information of co-/reverse transport related to ion selectivity. The selectivity of $\mathrm{CNTCC}$ to $\mathrm{Na}^{+}$and $\mathrm{Cl}^{-}$was determined by the $\mathrm{pH}$ gradient method. The kinetic curves obtained through this experiment are shown in Figure 4e,f. The fluorescence curve of AQPs has selectivity to $\mathrm{Na}^{+}$, and the fluorescence changes instead of being consistent with DOPC, which may be due to the gaps in the insertion of AQPs into the membrane, and the fluorescence changes. The fluorescence curve of CNTCC almost completely coincides with that of AQPs, indicating that it completely repels $\mathrm{Na}^{+}$and $\mathrm{Cl}^{-}$. 


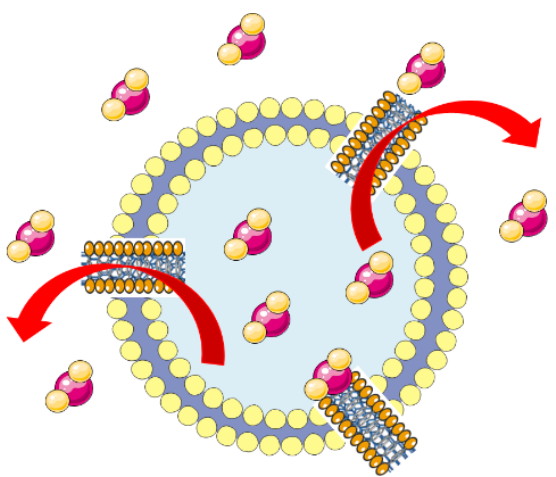

(a)

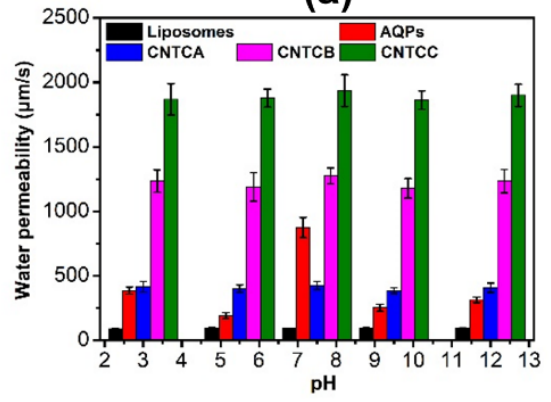

(c)

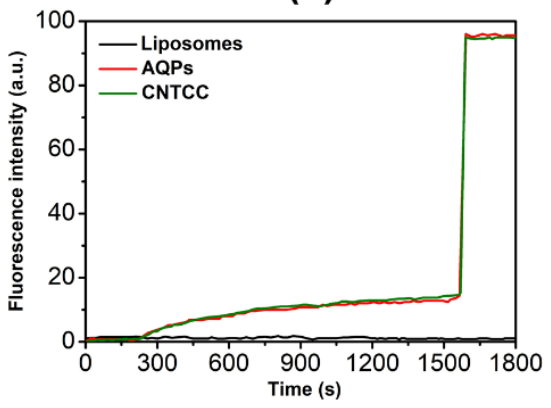

(e)

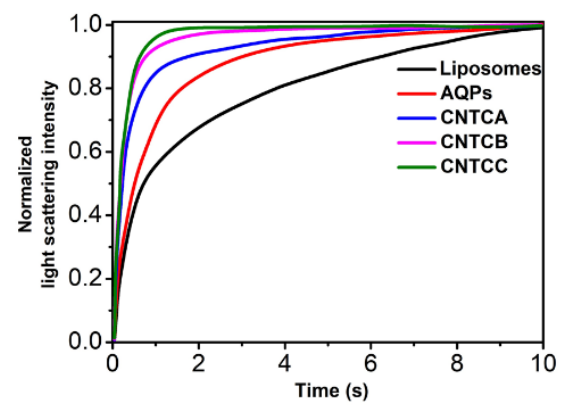

(b)

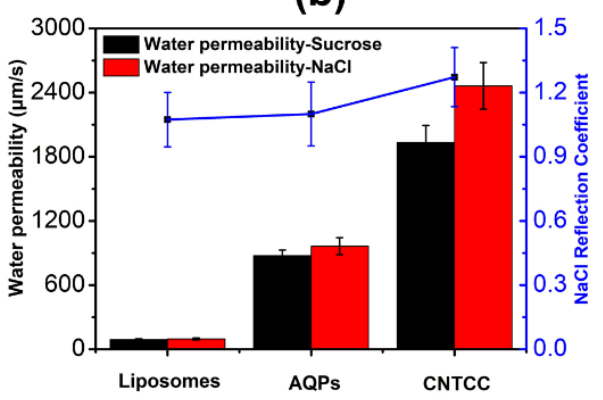

(d)

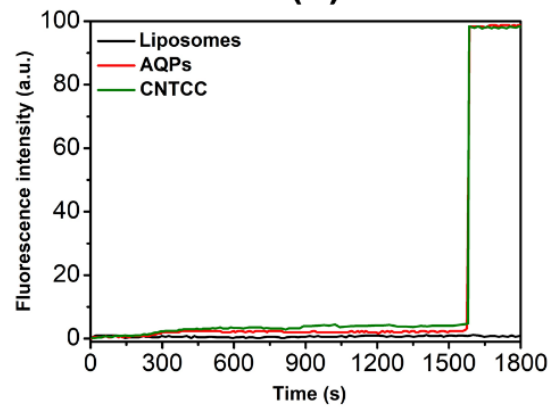

(f)

Figure 3. (a) shows that CNTC is embedded in liposomes (inset). The water molecules escape the interior of the liposomes through CNTC to reduce the applied permeability gradient. (b) The light scattering traces of different water channels under the same permeability gradient. (c) The water permeability values of AQPs and CNTC water channels were measured at different $\mathrm{pH}$. The water permeability of AQPs measured in our experiments is $875 \pm 78 \mu \mathrm{m} / \mathrm{s}$, which matches the literature value [15]. (d) Water permeability coefficient of DOPC liposome and proteoliposome (based on sucrose solute) and $\mathrm{NaCl}$ reflection coefficient (Equation (3)). Stop-flow experiment conditions: the osmotic pressure of $\mathrm{NaCl}$ and sucrose solutions were both $836 \mathrm{mosm} / \mathrm{L}$, while the vesicles contained HEPES buffer, and the osmotic pressure was $362 \mathrm{mosm} / \mathrm{L}$. Stop-flow measurement is performed by mixing equal volumes of draw solution and liposomes. Because of the chaotropic nature of $\mathrm{NaCl}$ [40], the water permeability measured by $\mathrm{NaCl}$ penetrant is greater than that measured with sucrose. (e,f) In 95:5 EYPC/EYPG liposomes (0.17 mM total lipid concentration), HPTS assay was used to determine the selectivity of different types of pore channels to $\mathrm{Na}^{+}$or $\mathrm{Cl}^{-}$. Conditions: by adding $50 \mu \mathrm{L} 0.5 \mathrm{M} \mathrm{NaOH}, 25 \mathrm{mM}$ HEPES, $100 \mathrm{mM} \mathrm{NaCl}$ inside the vesicle, $100 \mathrm{mM} \mathrm{NaCl}$ outside the vesicle, pH 7.0, base pulse. 


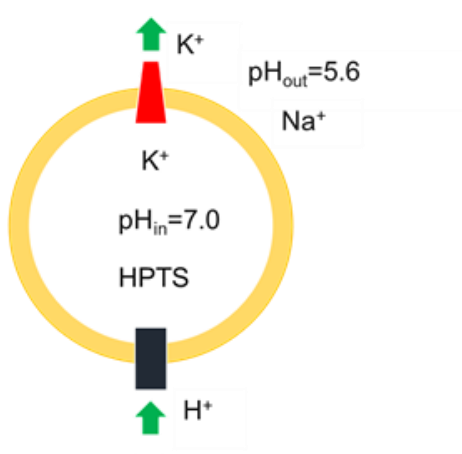

(a)

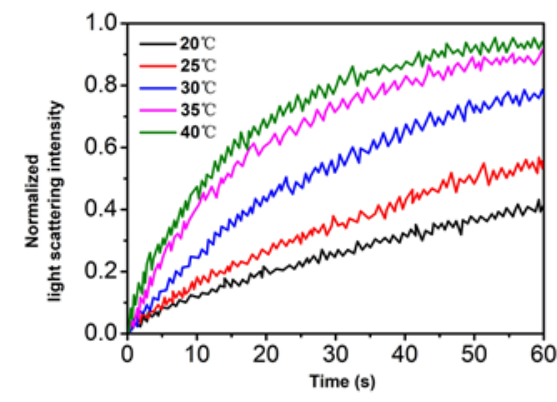

(c)

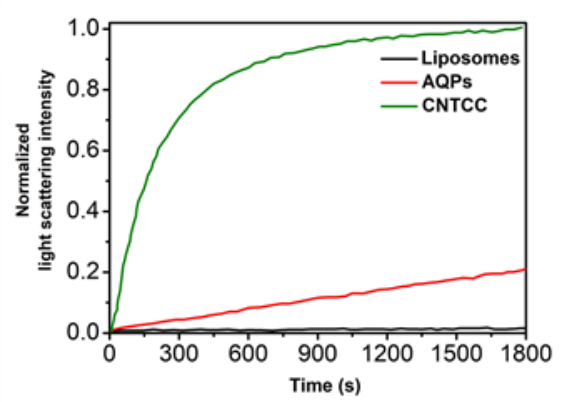

(b)

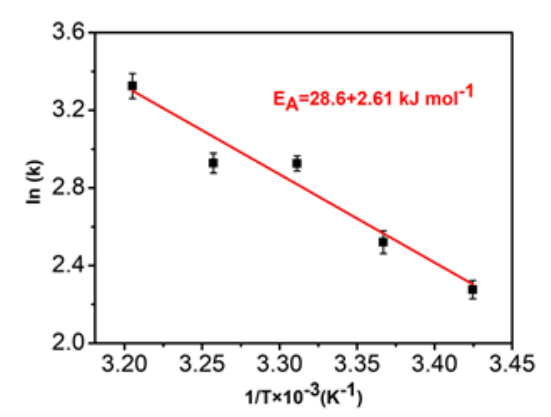

(d)

Figure 4. (a) Schematic diagram of $\mathrm{K}^{+}$and $\mathrm{H}^{+}$transmission in the channel caused by $\mathrm{pH}$ gradient changes. (b) After adding different water channels $(0.3 \mathrm{~mol} \%$ relative to liposomes) and valinomycin $(0.002 \mathrm{~mol} \%)$, the fluorescence intensity of vesicles $\left(\lambda_{\mathrm{ex}}=460 \mathrm{~nm}\right.$, $\left.\lambda_{\mathrm{em}}=510 \mathrm{~nm}\right)$ changes with time. The vesicles are filled with $\mathrm{NaCl}(100 \mathrm{mM})$ and HPTS (0.1 mM), buffered with HEPES $(10 \mathrm{mM})$ at $\mathrm{pH} 7.0$, and suspended in a $\mathrm{KCl}(100 \mathrm{mM})$ solution buffered to $\mathrm{pH}$ 7.6. (c) Changing in fluorescence intensity of CNTC-liposomes in the temperature range of $20-40{ }^{\circ} \mathrm{C}$. (d) The natural logarithm of the measured proton transmission rate $\mathrm{k}$, and a single exponential fitting is performed on the data drawn by the reciprocal of temperature. The Arrhenius activation energy $\left(E_{A}\right)$ was determined from the linear regression slope of the data.

\subsection{The Selectivity of CNTCs to Protons}

POMÉS et al. [41] proposed that if water molecules are connected to form a straight chain through hydrogen bonds, protons can be rapidly transported through the "Grotthus effect". In the natural AQPs pores, there are sites for binding water molecules at the narrow openings. The side chain of asparagine in the NPA region at the throat forms a new hydrogen bond with water molecules, causing the hydrogen bonds between the formed water molecules to rearrange, thereby breaking the hydrogen in the water line used by the rapid transport of protons in $\mathrm{H}_{3} \mathrm{O}^{+}$Bond, effectively preventing protons from passing through the water protein pore channel using the "Grotthus mechanism". As shown in Figure 4a, we inserted valinomycin and CNTCs together into liposomes to study CNTCs proton selectivity. Valinomycin has the characteristic of transferring $\mathrm{K}^{+}$from the high concentration side to the low concentration side [9]. When the external $\mathrm{pH}$ suddenly changes to $5.6, \mathrm{H}^{+}$will enter the liposome through the $\mathrm{CNTC}$ water channel, and $\mathrm{K}^{+}$will be transferred to the outside of the membrane through valinomycin to achieve ion balance inside and outside the membrane. After $\mathrm{H}^{+}$enters the membrane, the $\mathrm{pH}$ in the liposome cavity changes to trigger the change of the fluorescent dye HTPS in the cavity. The degree of change of the fluorescent dye reflects the degree of CNTC's ion selectivity. As shown in Figure $4 b$, the fluorescence change of CNTC is the most obvious, indicating that CNTC has the best selectivity to protons. The AQPs repels protons, because the flux of $\mathrm{H}^{+}$must be achieved through a continuous hydrogen bond network formed by water or hydroxyl groups [42]. The fluorescence change rate of CNTCC is greater than that of CNTCA and 
CNTCB. The length of CNTC not only affects its water permeability but also its proton selectivity. The transmission efficiency of protons has a great relationship with the length of CNTs water channel. The closer the length is to the lipid membrane and the higher the affinity, the higher the efficiency of proton transmission (See Figure S8 for details). The relationship between the transmission efficiency of protons and the size is consistent with the transmission efficiency of water. This is mainly because there are no amino acids, which play an important role within natural AQPs in the CNTC water channel, so it is impossible to block the transmission of hydrogen ions in the water chain [43]. We explored the temperature function in the range of $20-40^{\circ} \mathrm{C}$ to obtain the Arrhenius diagram, further confirming that CNTCC had high proton transfer characteristics. We obtained a linear curve of the proton diffusion rate constant (Figure 4c) with an activation energy value of $28.6 \pm$ $2.61 \mathrm{~kJ} / \mathrm{mol}$. In order to explain these values, we need to consider the proton transport mechanism. The movement of a proton along an isolated hydrogen-bonded water wire is essentially barrierless [41]; thus, the limiting factor for proton transport phenomena is the unfavorable free energy of transport of charged or polar species to the channel interior [44]. This energy includes a direct electrostatic component and a desolvation energy, both of which must be sufficiently compensated or minimized to allow rapid net transport [45]. The total free energy cost of protons passing through the hydrophobic center of CNTCs is predicted to be $42 \mathrm{~kJ} / \mathrm{mol}$ [46], which was also similar to the value of CNTCs measured by our experiments. Attractively, the energy barrier of proton transport in CNTCs was outstandingly larger than that in AQPs that we measured, namely $4.53 \mathrm{~kJ} / \mathrm{mol}$, which is consistent with previous reports [47]. The difference between proton transfer rate and activation energy report the structural difference between CNTCs and AQPs. The pores of the latter contain a variety of functional amino acids, which have the function of optimizing the channel to reduce the barrier to entry [45]. Near the contraction of the hydrophobic channel of AQPs, there are arginine $(\mathrm{R})$ and histidine $(\mathrm{H})$ residues in the AQPs monomer ring at positions R195 and H180, which provide the positive charge required for the water transport mechanism of AQPs [48], thereby repelling protons. The total $\mathrm{pI}$ value of histidine is 7.6, the pKa of its imidazole side chain is about 6.0, and the total pI value of arginine is 10.8 , and the pKa of its guanidyl side chain is 12.5. Therefore, due to the effect of protonation, the positive charge present on the residue will vary with $\mathrm{pH}$. This also explains that when the $\mathrm{pH}$ changes, the protonation state of the - $\mathrm{COOH}$ ending group of the amino acid residue changes, which may affect the water-pore interaction and the formation of the single water file structure of water molecules passing the AQPs channel. Therefore, the water transport efficiency of AQPs is affected, because the separation ability of AQPs stems from the formation of a single water molecule, which prevents proton translocation and relies on the positive charge at the H180 and R195 positions [49]. CNTCs not only have higher access barriers, but also their geometry promotes the formation of one-dimensional waterlines and enhances the transportation of Grotthuss, making the overall transportation rate higher than that of AQPs.

\section{Conclusions}

In summary, novel bionic water channel material CNTCs with high-affinity were prepared by cutting carbon nanotubes with precursor active agent on the surface. The water permeability and ion selectivity of CNTCs in lipid membrane system were also studied. Ultra-short CNTCs close to the thickness of the lipid membrane were synthesized in the process of ultrasound. As a precursor, phospholipids not only reduce the defect degree of CNTCs, but also improve the affinity of CNTCs. CNTCs have the characteristics of excellent dispersion, less defects, and high affinity, which improves the insertion efficiency of CNTCs in liposome system, thus improving the water permeability in whole system. The ultrashort and high-affinity CNTCs water channel completely rejects $\mathrm{Na}^{+}$and $\mathrm{Cl}^{-}$, and water permeability is $1936 \pm 123 \mu \mathrm{m} / \mathrm{s}$, which is 2.3 times that of natural AQPs. So far, the focus of the design of bionic water channels is to assemble into tubular structures and promote water conduction. Although the bionic water channel has made great breakthroughs in 
structure and function, it still lacks a variety of amino acids with specific functions similar to those in natural AQPs. In the future, it is necessary to find new methods to synthesize bionic water channels with special structures and sizes that can be precisely controlled at specific locations and a high similarity with AQPs. Nevertheless, these artificial bionic water channels with simpler structures and stronger mechanical properties are of great significance for studying the key structures and mechanisms of biological channel proteins and for further transplanting their functions into practical applications. With the research on artificial bionic water channels with rapid transport and selectivity, they will be more widely used in the fields of seawater desalination, nanofluidic device development, ultrasensitive biosensors, and biomedical diagnosis.

Supplementary Materials: The following are available online at https:/ / www.mdpi.com/2071-105 0/13/1/102/s1.

Author Contributions: Conceptualization: H.Z.; methodology: G.L.; software: G.L.; validation: G.L. and B.Z.; formal analysis: G.L.; investigation: J.L.; data curation: G.L.; writing-original draft preparation: G.L. and H.Z.; supervision: H.Z.; project administration: H.Z. All authors have read and agreed to the published version of the manuscript.

Funding: This research was funded by the National Natural Science Foundation of China, grant number 31800116.

Institutional Review Board Statement: Not applicable.

Informed Consent Statement: Not applicable.

Acknowledgments: This research was supported by The Key Laboratory of Water and Sediment Sciences and State Key Laboratory of Biobased Material and Green Papermaking. We appreciate all the participating researchers and institutions for their contributions to this work.

Conflicts of Interest: The authors declare no conflict of interest. The authors declare that they have no known competing financial interests or personal relationships that could have appeared to influence the work reported in this paper.

\section{References}

1. Werber, J.R.; Menachem, E. Permselectivity limits of biomimetic desalination membranes. Sci. Adv. 2018, 4, eaar8266. [CrossRef] [PubMed]

2. Li, X.; Wang, R.; Wicaksana, F. Preparation of high performance nanofiltration (NF) membranes incorporated with aquaporin Z. J. Membr. Sci. 2014, 450, 181-188. [CrossRef]

3. Li, X.; Loh, C.H.; Wang, R. Fabrication of a robust high-performance FO membrane by optimizing substrate structure and incorporating aquaporin into selective layer. J. Membr. Sci. 2016, 525, 257-268. [CrossRef]

4. Wang, M.; Wang, Z.; Wang, X. Layer-by-Layer Assembly of Aquaporin Z-Incorporated Biomimetic Membranes for Water Purification. Environ. Sci. Technol. 2015, 49, 3761-3768. [CrossRef] [PubMed]

5. Xie, W.; He, F.; Wang, B. An aquaporin-based vesicle-embedded polymeric membrane for low energy water filtration. J. Mater. Chem. A 2013, 1, 7592. [CrossRef]

6. Bowen, W.R. Biomimetic separations—Learning from the early development of biological membranes. Desalination 2006, 199, 225-227. [CrossRef]

7. Kumar, M.; Grzelakowski, M.; Zilles, J.; Clark, M.; Meier, W. Highly permeable polymeric membranes based on the incorporation of the functional water channel protein Aquaporin, Z. Proc. Natl. Acad. Sci. USA 2007, 104, 20719-20724. [CrossRef]

8. Shen, Y.X.; Si, W.; Erbakan, M. Highly permeable artificial water channels that can self-assemble into two-dimensional arrays. Proc. Natl. Acad. Sci. USA 2015, 112, 9810-9815. [CrossRef]

9. Hu, X.B.; Chen, Z.; Tang, G. Single-molecular artificial transmembrane water channels. J. Am. Chem. Soc. 2012, 134, 8384. [CrossRef]

10. Licsandru, E.; Kocsis, I.; Shen, Y.; Murail, S.; Legrand, Y.M.; Van Der Lee, A.; Tsai, D.; Baaden, M.; Kumar, M.; Barboiu, M. Salt-Excluding Artificial Water Channels Exhibiting Enhanced Dipolar Water and Proton Translocation. J. Am. Chem. Soc. 2016, 138, 5403-5409. [CrossRef]

11. Sanborn, J.R.; Chen, X.; Yao, Y.C. Carbon Nanotube Porins in Amphiphilic Block Copolymers as Fully Synthetic Mimics of Biological Membranes. Adv. Mater. 2018, 30, 1803355.1-1803355.9. [CrossRef] [PubMed]

12. Qin, J.; Zhu, B.; Liang, S. Impact of -C2H5 and -OH Functionalizations on the Water Flow Blockage in Carbon Nanotubes. J. Phys. Chem. C 2018, 122, 11807-11813. [CrossRef] 
13. Li, Q.; Li, X.; Ning, L. Hyperfast Water Transport through Biomimetic Nanochannels from Peptide-Attached (pR)-pillar[5]arene. Small 2019, 15, 1804678. [CrossRef] [PubMed]

14. Si, W.; Chen, L.; Hu, X.B. Selective artificial transmembrane channels for protons by formation of water wires. Angew. Chem. Int. Ed. 2015, 123, 12772-12776. [CrossRef]

15. Xie, W.; Low, J.W.J.; Armugam, A. Regulation of Aquaporin Z osmotic permeability in ABA tri-block copolymer. AIMS Biophys. 2015, 2, 381-397. [CrossRef]

16. Huelsenbeck, J.P. Bayesian inference of phylogeny and its impact on evolutionary biology. Science 2001, 294, 2310-2314. [CrossRef]

17. Li, H.; Chen, H.; Steinbronn, C. Enhancement of Proton Conductance by Mutations of the Selectivity Filter of Aquaporin-1. J. Mol. Biol. 2011, 407, 607-620. [CrossRef]

18. Janet, T.; Jaume, T. Can Stabilization and Inhibition of Aquaporins Contribute to Future Development of Biomimetic Membranes? Membranes 2015, 5, 352-368.

19. Barboiu, M.; Gilles, A. From Natural to Bioassisted and Biomimetic Artificial Water Channel Systems. Acc. Chem. Res. 2013, 46, 2814. [CrossRef]

20. Shen, Y.X.; Saboe, P.O.; Sines, I.T. Biomimetic membranes: A review. J. Membr. Sci. 2014, 454, 359-381. [CrossRef]

21. Noy, A.; Wanunu, M. A new type of artificial water channels. Nat. Nanotechnol. 2019, 15, 1-2. [CrossRef] [PubMed]

22. Hummer, G.; Rasaiah, J.C.; Noworyta, J.P. Water conduction through the hydrophobic channel of a carbon nanotube. Nature 2001, 414, 188-190. [CrossRef] [PubMed]

23. Upadhyay, R.K. Role of graphene/metal oxide composites as photocatalysts, adsorbents and disinfectants in water treatment: A review. RSC Adv. 2014, 4, 3823-3851. [CrossRef]

24. Hoenlein, W.; Kreupl, F.; Duesberg, G.S. Carbon nanotube applications in microelectronics. IEEE Trans. Compon. Packaging Technol. 2004, 27, 629-634. [CrossRef]

25. Cheng, X.; Zhou, W.; Li, P. Improving ultrafiltration membrane performance with pre-deposited carbon nanotubes/nanofibers layers for drinking water treatment. Chemosphere 2019, 234, 545-557. [CrossRef]

26. Tunuguntla, R.H.; Escalada, A.; Frolov, V.A. Synthesis, lipid membrane incorporation, and ion permeability testing of carbon nanotube porins. Nat. Protoc. 2016, 11, 2029-2047. [CrossRef]

27. Langecker, M. Synthetic Lipid Membrane Channels Formed by Designed DNA Nanostructures. Science 2012, $338,932-936$. [CrossRef]

28. Tunuguntla, R.H.; Chen, X.; Belliveau, A. High-Yield Synthesis and Optical Properties of Carbon Nanotube Porins. J. Phys. Chem. C 2017, 121, 3117-3125. [CrossRef]

29. Guido, P.; Micah, J.G.; Philippe, P. Competing mechanisms and scaling laws for carbon nanotube scission by ultrasonication. Proc. Natl. Acad. Sci. USA 2012, 109, 11599-11604.

30. Chew, H.B.; Moon, M.W.; Lee, K.R. Compressive dynamic scission of carbon nanotubes under sonication: Fracture by atomic ejection. In Proceedings of the Royal Society A: Mathematical. Phys. Eng. Sci. 2011, 2129, 1270-1289.

31. Bachilo, S.M.; Strano, M.S.; Kittrell, C.; Hauge, R.H.; Smalley, R.E.; Weisman, R.B. Structure-assigned optical spectra of singlewalled carbon nanotubes. Science 2002, 298, 1261-2366. [CrossRef] [PubMed]

32. Sun, X.; Zaric, S.; Daranciang, D.; Welsher, K.; Lu, Y.; Li, X.; Dai, H. Optical properties of ultrashort semiconducting single-walled carbon nanotube capsules down to sub-10 nm. J. Am. Chem. Soc. 2008, 130, 6551-6555. [CrossRef] [PubMed]

33. Opilik, L.; Bauer, T.; Schmid, T.; Stadler, J.; Zenobi, R. Nanoscale chemical imaging of segregated lipid domains using tipenhanced Raman spectroscopy. Phys. Chem. Chem. Phys. 2011, 13, 9978-9981. [CrossRef] [PubMed]

34. Stochastic transport through carbon nanotubes in lipid bilayers and live cell membranes. Nature 2014, 514, 612-615. [CrossRef] [PubMed]

35. Zuo, G.; Shen, R.; Ma, S. Transport properties of single-file water molecules inside a carbon nanotube biomimicking water channel. ACS Nano 2010, 4, 205. [CrossRef]

36. Sheng, J.; Zhu, Q.; Zeng, X. Promotion of Water Channels for Enhanced Ion Transport in 14 nm Diameter Carbon Nanotubes. ACS Appl. Mater. Interfaces 2017, 9, 11009. [CrossRef]

37. Murata, K.; Kaoru, M.; Hirai, T. Structural determinants of water permeation through aquaporin-1. Nature 2000, 407, 599-605. [CrossRef]

38. Kitchen, P.; Conner, A.C. Control of the Aquaporin-4 Channel Water Permeability by Structural Dynamics of Aromatic/Arginine Selectivity Filter Residues. Biochemistry 2015, 54, 6753-6755. [CrossRef]

39. Montesarchio, D.; Coppola, C.; Boccalon, M. Carbohydrate-based synthetic ion transporters. Carbohyd. Res. 2012, 356, 62-74. [CrossRef]

40. Tobias, D.J.; Hemminger, J.C. Getting Specific about Specific Ion Effects. Science 2008, 319, 1197-1198. [CrossRef]

41. Pomès, R.; Roux, B. Free energy profiles for H+ conduction along hydrogen-bonded chains of water molecules. Biophys. J. 1998, 75, 33-40. [CrossRef]

42. Groot, B.L.D.; Engel, A.; Grubmüller, H. The Structure of the Aquaporin-1 Water Channel: A Comparison between Cryo-electron Microscopy and X-ray Crystallography. J. Mol. Biol. 2003, 325, 485-493. [CrossRef]

43. Verkman, A.S.; Mitra, A.K. Structure and function of aquaporin water channels. Am. J. Physiol. Renal Phys. 2000, 278, F13. [CrossRef] [PubMed] 
44. Braun-Sand, S.; Burykin, A.; Chu, Z.T. Realistic simulations of proton transport along the gramicidin channel: Demonstrating the importance of solvation effects. J. Phys. Chem. B 2005, 109, 583. [CrossRef] [PubMed]

45. Wraight, C.A. Chance and design-Proton transfer in water, channels and bioenergetic proteins. Biol. Biol. Acta 2006, 1757, 886-912. [CrossRef]

46. Dellago, C.; Hummer, G. Kinetics and Mechanism of Proton Transport across Membrane Nanopores. Phys. Rev. Lett. 2006, 97, 245901. [CrossRef] [PubMed]

47. Tunuguntla, R.; Allen, F.; Kim, K. Ultrafast proton transport in sub-1-nm diameter carbon nanotube porins. Biophys. J. 2016, 110, 338a. [CrossRef]

48. Chen, H.; Wu, Y.; Voth, G.A. Origins of Proton Transport Behavior from Selectivity Domain Mutations of the Aquaporin-1 Channel. Biophys. J. 2006, 90, L73-L75. [CrossRef]

49. Nemeth-Cahalan, K.L.; Hall, J.E. pH and Calcium Regulate the Water Permeability of Aquaporin 0. J. Biol. Chem. 2000, 275, 6777-6782. [CrossRef] 